\title{
ASYMPTOTIC BEHAVIOR OF SOLUTIONS TO SOME $n$ TH ORDER LINEAR DIFFERENTIAL EQUATIONS ${ }^{1}$
}

\author{
I. NORMAN KATZ
}

Let $L u \equiv u^{(n)}+\sum_{j=0}^{n-2} a_{j}(t) u^{(j)}$, where $a_{j}(t), j=0, \cdots, n-2$, are continuous on $\left[t_{0}, \infty\right)$, and let $z_{j}(t), j=1, \cdots, n$, be linearly independent solutions to

$$
L z=0 .
$$

We study the behavior as $t \rightarrow \infty$ of solutions to

$$
L u=\sum_{j=0}^{n-2} g_{j}(t) u^{(j)}
$$

where $g_{j}(t), j=0, \cdots, n-2$, are continuous on $\left[t_{0}, \infty\right)$, in terms of the known functions $z_{1}(t), \cdots, z_{n}(t)$. Specifically, we seek conditions in order that for each solution $u(t)$ of (2), there exist constants $A_{j}$, $j=1, \cdots, n$, such that

$$
u^{(i)}(t)=A_{1}(t) z_{1}^{(i)}(t)+\cdots+A_{n}(t) z_{n}^{(i)}(t), \quad i=0, \cdots, n-1,
$$

and

$$
\lim _{t \rightarrow \infty} A_{j}(t)=A_{j}, \quad \int_{t_{0}}^{\infty}\left|A_{j}^{\prime}(t)\right| d t<\infty, \quad j=1, \cdots, n,
$$

hold; and that for each choice of $A_{j}$ there exists a solution $u(t)$ of (2) satisfying (3) and (4). For bounded functions $z_{j}^{(k-1)}(t), j=1, \cdots, n$, $k=1, \cdots, n$, this is stronger than the case where (1) and (2) are asymptotically equivalent, i.e., for each solution $u(t)$ of $(2)$ there is a solution $z(t)$ of (1) such that $\left|u^{(i)}(t)-z^{(i)}(t)\right| \rightarrow 0$ as $t \rightarrow \infty, i=0, \cdots$, $n-1$, and conversely (see [3]).

Denote by $W(t)=W\left(z_{1}(t), \cdots, z_{n}(t)\right)$ the Wronskian of $z_{j}(t)$, $j=1, \cdots, n$, and let $W_{j}(t), j=1, \cdots, n$, be the determinant obtained by replacing all elements in the $j$ th column of $W(t)$ by zero except for the element in the $n$th row which is replaced by 1 . Let

$$
y(t)=\max _{k=1, \cdots, n}\left|\sum_{i=0}^{n-2} g_{i}(t) z_{k}^{(i)}(t)\right| .
$$
1968.

Presented to the Society, January 24, 1969; received by the editors August 12,

1 This work was supported in part by NASA Grant NLG26-008-006. 
We prove the following theorem.

THEOREM. Given arbitrary constants $A_{j}, j=1, \cdots, n$, there exists a solution to (2) which can be written in the form (3) with $A_{j}(t), j=1, \cdots$, $n$, satisfying (4) if and only if

$$
\sum_{j=1}^{n} \int_{t_{0}}^{\infty}\left|W_{j}(t)\right| y(t) d t<\infty .
$$

Also, every solution to (2) can be written in the form (3) with $A_{j}(t)$ satisfying (4) (for some finite $A_{j}, j=1, \cdots, n$ ) if and only if (5) is satisfied.

REMARK 1 . Our theorem is essentially a characterization of the method of variation-of-constants $[1$, p. 87], applied to (2) when the general solution to (1) is known. The absence of the $z^{(n-1)}$ term in (1) and the $u^{(n-1)}$ term in (2) implies that $W\left(z_{1}(t), \cdots, z_{n}(t)\right)$ $=W\left(z_{1}\left(t_{0}\right), \cdots, \quad z_{n}\left(t_{0}\right)\right)=W\left(t_{0}\right) \neq 0 \quad$ and $W\left(u_{1}(t), \cdots, \quad u_{n}(t)\right)$ $=W\left(u_{1}\left(t_{0}\right), \cdots, u_{n}\left(t_{0}\right)\right) \neq 0$ when $u_{1}(t), \cdots, u_{n}(t)$ are linearly independent solutions to (2), and this is essential to our proof of the theorem.

When $n=2,\left|W_{1}(t)\right|=\left|z_{2}(t)\right|,\left|W_{2}(t)\right|=\left|z_{1}(t)\right|$, and it is easily verified that (5) is equivalent to

$$
\int_{t_{0}}^{\infty}\left|g_{0}(t)\right| \max \left[\left|z_{1}(t)\right|^{2},\left|z_{2}(t)\right|^{2}\right] d t<\infty .
$$

This condition was shown to be sufficient by Trench [2]. (Trench [2] does not state that $\int_{t_{0}}^{\infty}\left|A_{j}^{\prime}(t)\right| d t<\infty, j=1,2$, when (6) is satisfied but this follows easily from his proof.) It follows from our theorem that (6) is also necessary if all solutions to (2) are required to satisfy $\int_{t_{0}}^{\infty}\left|A_{j}^{\prime}(t)\right| d t<\infty, j=1,2$. Other related results for arbitrary $n$ are obtained in [3], [4], [5], and [6].

REMARK 2 . In the special case when $a_{j}(t) \equiv 0, j=0, \cdots, n-2,(2)$ becomes

$$
u^{(n)}=\sum_{i=0}^{n-2} g_{i}(t) u^{(i)} .
$$

Now we have $z_{k}(t)=t^{k-1}, k=1, \cdots, n$, and it is easily shown that $W_{j}(t)=O\left(t^{n-j}\right)$ as $t \rightarrow \infty, j=1, \cdots, n$. From our theorem it follows that if $\int_{t_{0}}^{\infty}\left|g_{i}(t)\right| t^{2 n-2-i} d t<\infty, i=0, \cdots, n-2$, then every solution to (7) can be written in the form $u(t)=\sum_{\mathbf{k}=1}^{n} A_{k}(t) t^{k-1}, u^{(i)}(t)=$ $\sum_{k=i+1}^{n}(k-1) \quad \cdots \quad(k-i) A_{k}(t) t^{k-1-i}, \quad i=1, \cdots, \quad n-1$ where $\lim _{t \rightarrow \infty} A_{k}(t), k=1, \cdots, n$, may be prescribed. 
Proof. Suppose $u_{k}(t), k=1, \cdots, n$, are linearly independent solutions to (2) which can be written in the form

$$
u_{k}^{(i)}(t)=\sum_{j=1}^{n} A_{j k}(t) z_{j}^{(i)}(t), \quad i=0, \cdots, n-1,
$$

with $\int_{t_{0}}^{\infty}\left|A_{j k}^{\prime}(t)\right| d t<\infty, j=1, \cdots, n, k=1, \cdots, n$, (which implies that $\lim _{t \rightarrow \infty} A_{j k}(t)=A_{j k}$ exists and is finite). Let the matrix $A(t)$ $\equiv\left[A_{j k}(t)\right]$ and let the matrix $A \equiv\left[A_{j k}\right]$. Then

$$
W\left(u_{1}(t), \cdots, u_{n}(t)\right)=W\left(z_{1}(t), \cdots, z_{n}(t)\right) \operatorname{det} A(t) .
$$

Since $W\left(u_{1}(t), \cdots, u_{n}(t)\right)=W\left(u_{1}\left(t_{0}\right), \cdots, u_{n}\left(t_{0}\right)\right) \neq 0$ and $W\left(z_{1}(t), \cdots, z_{n}(t)\right)=W\left(t_{0}\right) \neq 0$ it follows that $\operatorname{det} A(t)=\operatorname{det} A \neq 0$. Now given arbitrary constants $A_{j}, j=1, \cdots, n$, let $B_{k}, k=1, \cdots, n$, be determined from $\sum_{k=1}^{n} A_{j k} B_{k}=A_{j}, j=1, \cdots, n$, and let $A_{j}(t)$ $=\sum_{k=1}^{n} A_{j k}(t) B_{k}$. Then $\lim _{t \rightarrow \infty} A_{j}(t)=A_{j}$ and $\int_{t_{0}}^{\infty}\left|A_{j}^{\prime}(t)\right| d t<\infty$, $j=1, \cdots, n$. Also

$$
\begin{aligned}
u^{(i)}(t) & =\sum_{k=1}^{n} B_{k} u_{k}^{(i)}(t)=\sum_{j=1}^{n}\left(\sum_{k=1}^{n} A_{j k}(t) B_{k}\right) z_{j}^{(i)}(t) \\
& =\sum_{k=1}^{n} A_{j}(t) z_{j}^{(i)}(t), \quad i=0, \cdots, n-1
\end{aligned}
$$

is in the form (3) and satisfies (2). Thus, if we show that (5) is a necessary and sufficient condition for the existence of $n$ linearly independent solutions to (2) which can be written in the form (3) satisfying (4), this will show that a solution $u(t)$ to (2) exists which can be written in the form (3) when $A_{j}, j=1, \cdots, n$, are arbitrary constants. Also, any solution to (2) can be written as a linear combination of $n$ linearly independent solutions. Therefore, we need only to prove the theorem for linearly independent solutions $u_{k}(t), k=1$, $\cdots, n$.

Let $u(t)=\sum_{j=1}^{n} A_{j}(t) z_{j}(t)$ be a solution to (2) satisfying (3). Then it follows that

$$
\begin{aligned}
\sum_{j=1}^{n} A_{j}^{\prime}(t) z_{j}^{(i)}(t) & =0, \quad i=0, \cdots, n-2 \\
\sum_{j=1}^{n} A_{j}^{\prime}(t) z_{j}^{(n-1)}(t) & =\sum_{i=0}^{n-2} g_{i}(t) u^{(i)}(t) \\
& =\sum_{j=1}^{n}\left(\sum_{i=0}^{n-2} g_{i}(t) z_{j}^{(i)}(t)\right) A_{j}(t) .
\end{aligned}
$$


Conversely, if $A_{j}(t), j=1, \cdots, n$, satisfy (9) then $u(t)$ is a solution to (2).

We now show that if $(5)$ is satisfied, and $A_{j}(t), j=1, \cdots, n$, satisfy (9), then

$$
\int_{t_{0}}^{\infty}\left|A_{j}^{\prime}(t)\right| d t<\infty
$$

(which implies also that $\lim _{t \rightarrow \infty} A_{j}(t)$ exists and is finite). From (9) we have

$$
\begin{array}{r}
A_{j}^{\prime}(t)=\left(W_{j}(t) / W\left(t_{0}\right)\right) \sum_{k=1}^{n} A_{k}(t) \sum_{i=0}^{n-2} g_{i}(t) z_{k}^{(i)}(t), \quad j=1, \cdots, n, \\
A_{j}(t)=A_{j}\left(t_{0}\right)+\left(1 / W\left(t_{0}\right)\right) \int_{t_{0}}^{t} \sum_{k=1}^{n} A_{k}(s) W_{j}(s) \sum_{i=0}^{n-2} g_{i}(s) z_{k}^{(i)}(s) d s, \\
j=1, \cdots, n,
\end{array}
$$

and

$$
\begin{aligned}
&\left|A_{j}(t)\right| \leqq\left|A_{j}\left(t_{0}\right)\right|+\left(1 /\left|W\left(t_{0}\right)\right|\right) \\
& \cdot \int_{t_{0}}^{t} \sum_{k=1}^{n}|| A_{k}(s)\left|W_{j}(s)\right|\left|\sum_{i=0}^{n-2} g_{i}(s) z_{k}^{(i)}(s)\right| d s \\
& \leqq\left|A_{j}\left(t_{0}\right)\right|+\left(1 /\left|W\left(t_{0}\right)\right|\right) \int_{t_{0}}^{t} \sum_{k=1}^{n}\left|A_{k}(s)\right|\left|W_{j}(s)\right| y(s) d s \\
& \quad j=1, \cdots, n .
\end{aligned}
$$

Summing over $j$ we obtain

$$
\begin{aligned}
\sum_{j=1}^{n}\left|A_{j}(t)\right| \leqq & \sum_{j=1}^{n}\left|A_{j}\left(t_{0}\right)\right|+\left(1 /\left|W\left(t_{0}\right)\right|\right) \\
& \cdot \int_{t_{0}}^{t}\left(\sum_{k=1}^{n}\left|A_{k}(s)\right|\right)\left(\sum_{j=1}^{n}\left|W_{j}(s)\right| y(s)\right) d s .
\end{aligned}
$$

Using Bellman's lemma $[7$, p. 35], we have

$$
\begin{aligned}
\sum_{j=1}^{n}\left|A_{j}(t)\right| \leqq\left(\sum_{j=1}^{n}\left|A_{j}\left(t_{0}\right)\right|\right) \exp \left(\left(1 /\left|W\left(t_{0}\right)\right|\right)\right. \\
\left.\cdot \int_{t_{0}}^{t} \sum_{j=1}^{n}\left|W_{j}(s)\right| y(s) d s\right) .
\end{aligned}
$$

Since (5) is satisfied, the integral in (11) is convergent as $t \rightarrow \infty$ which 
implies that $A_{j}(t), j=1, \cdots, n$ are bounded. This used in (10) gives

$$
\begin{aligned}
& \int_{t_{0}}^{t}\left|A_{j}^{\prime}(s)\right| d s \leqq\left(1 /\left|W\left(t_{0}\right)\right|\right) \int_{t_{0}}^{t} \sum_{k=1}^{n}\left|A_{k}(s)\right|\left|W_{j}(s)\right| \\
& \cdot\left|\sum_{i=0}^{n-2} g_{i}(s) z_{k}^{(i)}(s)\right| d s \\
& \leqq\left(1 /\left|W\left(t_{0}\right)\right|\right) \int_{t_{0}}^{t} \sum_{k=1}^{n}\left(\left|A_{k}(s)\right|\right)\left|W_{j}(s)\right| y(s) d s, \\
& j=1, \cdots, n,
\end{aligned}
$$

which shows that $\int_{t_{0}}^{\infty}\left|A_{j}^{\prime}(t)\right| d t<\infty, j=1, \cdots, n$. Now let $A_{j k}(t)$ be the solution of (9) with $A_{j k}\left(t_{0}\right)=\delta_{j k}, j=1, \cdots, n, k=1, \cdots, n$, and let

$$
u_{k}(t)=\sum_{j=1}^{n} A_{j k}(t) z_{j}(t), \quad k=1, \cdots, n .
$$

Then $u_{k}(t), k=1, \cdots, n$, satisfies (2),

$$
u_{k}^{(i)}(t)=\sum_{j=1}^{n} A_{j k}(t) z_{k}^{(i)}(t), \quad i=0, \cdots, n-1,
$$

is in the form (3) with $A_{j k}(t)$ satisfying (4), and from (8) it follows that $u_{k}(t), k=1, \cdots, n$, are linearly independent. This completes the proof of the sufficiency of (5).

To prove the necessity of (5), assume that

$$
u_{k}(t)=\sum_{j=1}^{n} A_{j k}(t) z_{j}(t), \quad k=1, \cdots, n,
$$

are linearly independent solutions to (2) which satisfy (3) and (4). Then it follows from (8) that $\operatorname{det} A(t)=\operatorname{det} A\left(t_{0}\right) \neq 0$. Therefore, from (10), $A(t)$ is a fundamental matrix for the matrix equation

$$
A^{\prime}=Z(t) A
$$

where the matrix

$$
\begin{aligned}
Z(t) \equiv\left(1 /\left(W\left(t_{0}\right)\right)\right)\left[W_{j}(t)\right. & \left.\sum_{i=0}^{n-2} g_{i}(t) z_{k}^{(i)}(t)\right], \\
& j=1, \cdots, n, k=1, \cdots, n .
\end{aligned}
$$

Thus 
$Z(t)=A^{\prime}(t) A^{-1}(t)=(1 / \operatorname{det} A(t)) A^{\prime}(t) B(t)=\left(1 / \operatorname{det} A\left(t_{0}\right)\right) A^{\prime}(t) B(t)$,

where the elements of $B(t)$ are sums of products of the $A_{j k}(t)$. Since by assumption the $A_{j k}(t)$ approach finite limits as $t \rightarrow \infty$ the same is true for the elements of $B(t)$. Since by assumption

$$
\int_{t_{0}}^{\infty}\left|A_{j k}^{\prime}(t)\right| d t<\infty, \quad j=1, \cdots, n, k=1, \cdots, n,
$$

it now follows that the elements of $Z(t)$ are absolutely integrable over $\left[t_{0}, \infty\right)$, that is

$$
\int_{t_{0}}^{\infty}\left|W_{j}(t)\right|\left|\sum_{i=0}^{n-2} g_{i}(t) z_{k}^{(i)}(t)\right| d t<\infty, \quad j=1, \cdots, n, k=1, \cdots, n .
$$

It is easily verified that this implies (5).

\section{REFERENCES}

1. E. A. Coddington ana N. Levinson, Theory of ordinary differential equations, McGraw-Hill, New York, 1955.

2. W. F. Trench, On the asymptotic behavior of solutions to second order linear differential equations, Proc. Amer. Math. Soc. 14 (1963), 12-14.

3. F. Brauer, Asymptotic equivalence and asymptotic behavior of linear systems, Michigan Math. J. 9 (1962), 33-43.

4. T. G. Hallam, Asymptotic behavior of the solutions of an nth order nonhomogeneous ordinary differential equation, Trans. Amer. Math. Soc. 122 (1966), 177-194.

5. P. Locke, On the asymptotic behavior of solutions of an nth order nonlinear equation, Proc. Amer. Math. Soc. 18 (1967), 383-390.

6. F. Brauer and J. S. W. Wong, On the asymptotic behavior of perturbed linear systems, J. Differential Equations (to appear).

7. R. Bellman, Stability theory of differential equations, McGraw-Hill, New York, 1953.

\section{WASHINGTON UNIVERSITY}

